\title{
The Influence of Telemedicine and Compassionate Care on the Quality of Life and Mental Health of Patients with Epilepsy in Northeastern China During the COVID-19 Crisis
}

\author{
Deshuang Tao ${ }^{1,2, *}$ \\ Tangwu Zhong ${ }^{2} *$ \\ Juli Wang'
}

'Epilepsy Department of Jiamusi Central Hospital, Jiamusi City, Heilongjiang Province, 154002, People's Republic of China; ${ }^{2}$ Jiamusi University, Jiamusi City, Heilongjiang Province, I54007, People's Republic of China

*These authors contributed equally to this work
Correspondence: Juli Wang

Tel +86I8724540I30

Emailwjll9898I@I63.com
Purpose: To understand the influence of telemedicine and compassionate care on the quality of life and mental health of patients with epilepsy (PWE) in northeastern China during the COVID-19 crisis.

Patients and Methods: Physicians in the epilepsy department conducted a questionnaire survey on PWE on February 2020. The Quality Of Life In Epilepsy-31 (QOLIE-31), Generalized Anxiety Disorder 7-Item Scale (GAD-7) and Patient Health Questionnaire-9 (PHQ-9) were used. The intervention (IG) group received compassionate care and follow-up through telemedicine equipment every week, while the nonintervention (NIG) group did not receive. The questionnaire survey was conducted again three month later.

Results: Ninety patients were recruited: mean age $39.91 \pm 15.57$ in the IG, $37.39 \pm 11.69$ in the NIG, 46 (51.1\%) were men. Twenty patients had difficulty in purchasing antiepileptic drugs (AEDs). Seven patients reported seizure in the last 1 month. Only 1 patient $(2.2 \%)$ consulted the emergency department. Up to 84 patients' lives were affected. Fifteen $(33.3 \%)$ of the IG and $20(44.4 \%)$ of the NIG patients stated that their family income had decreased, and among them, $13(28.9 \%)$ in the IG group and $10(22.2 \%)$ in the NIG group stated that they were unemployed. 3 months later, the interaction between groups and time of QOLIE-31 was significant, $\mathrm{F}(1,88)=16.996, \mathrm{p}<0.001$; the interaction between group and time on the PHQ9 was significant, $\mathrm{F}(1,88)=14.992, \mathrm{p}<0.001$; the interaction between group and time on the QAD-7 was significant, $F(1,88)=6.026, \mathrm{p}<0.001$.

Conclusion: Our study found that during the COVID-19 outbreak, when patients were in a lockdown, telemedicine and compassionate care were effective and successful in managing PWE in northeastern China. It is a valid method to decrease anxiety and depression and improve the patients' quality of life. Further research is necessary about compassionate care methods for PWE.

Keywords: telemedicine, compassionate care, QOLIE-31, GAD-7, COVID-19, epilepsy

\section{Introduction}

In December 2019, an epidemic of acute respiratory infections broke out in China. The identified infective agent is a novel coronavirus, and the disease caused by this agent is called "coronavirus disease 2019" (COVID-19). ${ }^{1}$ To control the COVID-19 pandemic, China has carried out strict city isolation. Up to March 26, 2020, at least 82,078 cases of COVID-19 had been confirmed in China, with 3298 known deaths, representing a fatality rate of approximately $4 \% .^{2}$ 
People with epilepsy (PWE) faced the consequences of the pandemic. ${ }^{3}$ Mental health issues are common in PWE. Anxiety may particularly be heightened from additional concerns about the pandemic. ${ }^{4}$ Not all cities in northeastern China have epilepsy centers. The epilepsy center of our hospital serves adult and child PWE in nearly 10 areas in northeastern China. PWE regularly go to our hospital to buy antiepileptic drugs (AEDs) and regularly recheck their electroencephalogram (EEG). When the epidemic broke out, many patients did not have enough AEDs, and their scheduled rechecks were cancelled. Most importantly, no one could give an end time. Thus, the epidemic led to great changes in people's lives, including PWE. We received many calls every day, asking about their condition, how to buy AEDs, and the next treatment plan. To alleviate the impact of the patients' negative emotions, we actively looked for ways to help them.

In southwestern China, during the COVID-19 outbreak, health care professionals focused on seizure control and the mental health of PWE, and they advised PWE not to pay too much attention to media coverage of the pandemic, such as by distracting themselves with other activities. PWE with severe psychological distress should consult a psychiatrist. ${ }^{5}$ In Malaysia, a study reported that health care services discouraged PWE-adjusted AEDs because this was associated with seizure worsening, and some patients who experienced seizure improvement may have attributed this to good sleep and less work stress. ${ }^{6}$ A self-selected cohort study, most patients were satisfied with remote audio-only telephone consultations during the global COVID-19 pandemic, and Telemedicine is likely to become an integral part of routine care for chronic neurological illnesses hereafter. ${ }^{7}$

Telemedicine is the delivery of health care and the exchange of health-care information across distances. ${ }^{89}$ Telemedicine is practiced well in industrialized countries but it is somewhat deficient in developing countries. ${ }^{10,11}$ The lack of neurologists in rural settings has resulted in significant financial and logistical challenges for PWE. The restrictions have made outpatient face-to-face consultations impossible during the COVID-19 pandemic, and many neurologists have started using telemedicine to manage their patients. ${ }^{9}$ The Australian Epilepsy Project has transitioned to tele-neuropsychology, which enables largescale neuropsychological research during COVID-19. ${ }^{12}$ The American Academy of Neurology (AAN) provided a practical framework for translating quality care in epilepsy guidelines into a virtual care environment by telehealth. ${ }^{13}$ A German epilepsy center assessed acceptance and appreciation of telemedicine, and overall $82 \%$ of the participants were satisfied. ${ }^{14}$

Compassion is extolled as a cornerstone of quality health care by patients, families, and clinicians. ${ }^{15}$ The importance of compassion is attested to by patients and their families, who have consistently ranked features of compassion among their greatest health care needs. ${ }^{16}$ Compassion has been recognized as a marker and medium of spiritual care and an ameliorator of suffering. ${ }^{17}$ A compassionate relationship is marked by meaning, a genuine sense of caring for the patient, and a willingness to provide support. ${ }^{18}$ Although compassionate care seems intuitive, there are still incidents of substandard care. Health care educators face challenges developing and sustaining these core competencies with students because they exhibit fewer caring behaviors and less empathy. ${ }^{19}$ Clinicians and patients also have different versions of compassionate care. Despite many challenges, clinicians have at their disposal a myriad of tools that can be used to express compassion and empathy to their patients during the global COVID-19 pandemic. $^{20-22}$ However, there is no prior report of using telemedicine and compassionate care to manage PWE in northeastern China during the COVID-19 pandemic. To evaluate the influence of telemedicine and compassionate care on the quality of life and mental health of patients with epilepsy during the emergency determined by the COVID-19 pandemic, we designed the study.

\section{Patients and Methods}

\section{Study Participants}

On February 15 to 25, 2020, four physicians from the epilepsy department of Jiamusi Central Hospital who did not know about the experiment searched inpatients from January 2018 to January 2020 through the electronic medical record query system. Inclusive criteria: Patients with focal epilepsy (FE); Oxcarbazepine monotherapy; Regular treatment for at least one year; Consent to participate in the questionnaire survey; No mental illness; No hearing and speech disorders; Older than 14 years old; Currently living in northern China. Exclusion criteria: Unwilling or unable to cooperate; Younger than 14 years old; Patients with mental disorders; Patients with irregular treatment or in the process of drug reduction; Generalized epilepsy (GE); Currently does not live in northern China. 
Diagnosis of FE and GE were made according to the diagnostic criteria of the International League Against Epilepsy (ILAE). The two main epilepsy types differ by the definition of their seizure semiology: FE is characterized by focal seizures originating from one cerebral hemisphere; GE is characterized by seizures involving both cerebral hemispheres. ${ }^{23}$ First, the clinician inquired about the patient's history in detail. If it is a relatively clear focal seizure, combined with focal EEG abnormalities and focal ictal semiology, it was included in the diagnosis of FE. If PWE could not ensure the ictal semiology, we diagnosed FE by finding focal EEG abnormalities during the ictal and interictal periods. If PWE were unable to identify the ictal semiology and there were no focal EEG abnormalities, the case was submitted to our evaluation team for discussion, and any cases for which we were unable to make a clear diagnosis were excluded.

This experiment passed the examination standard of the ethics committee of Jiamusi Central Hospital. All patients included gave oral consent for participation. Before the questionnaires, the purpose of the assessment, the time required, and the confidentiality principle of the patient's information were provided to the patients.

Compassionate care: The intervention group received compassionate care every week, while the nonintervention group did not receive any compassionate care. Our compassionate care mainly included three main parts. The first part is the descriptive questions, which include asking the patients about their diet, sleep, mood, home activities, home sports, and family relationships during the last week. The patients were required to use four answers to respond: good, general, poor and especially poor. The second part was the selective questions, including whether they were worried about the epidemic situation, whether they were worried about their economic situation, whether they were worried about being fired, whether they had any quarrels with others, whether they paid attention to maintaining their hygiene and image, whether they went out, and whether they had cooked a delicious food they missed. The patients were required to use two answers to respond: yes or no. When we found that the patient had pessimistic or negative answers to the selective questions, we chatted with the patient through encouragement or enlightenment. The last part included whether the AEDs were sufficient, whether there were other AEDs available locally, whether there were any seizures in the last week, whether there were adverse reactions to the oral AEDs in the last week, whether oral AEDs were regularly taken in the last week, whether there were other physical discomforts in the last week, such as diarrhea and constipation. Other questions included have you wanted to do nothing or even thought about committing suicide this week, did you pay attention to the increasing numbers of epidemic cases and the reports of fighting against the epidemic situation, and did you watch television or your phone almost constantly. The last part deals with telemedicine. We chose video calls or phone calls to communicate with the patients in different situations. The situations that needed video calls included adverse drug reactions and emotional disorders. Other situations were generally managed with phone calls.

The questionnaire started after the patient agreed and was completed within 20 minutes. The original Quality Of Life In Epilepsy-31 (QOLIE-31) includes 30 items clustered in seven multi-item scales and one single item on overall health. Multi-item scales are centered on the following domains: seizure worry; overall quality of life; emotional well-being; energy/fatigue; cognitive functioning; medication effects; and social functioning. A scoring system is available for each item and is calculated for each scale. The raw values were converted to $0-100$ scores, with higher values reflecting better quality of life. The scores are calculated for each domain, and the overall score is obtained by using a weighted average of the multiitem scale scores. ${ }^{24}$ Generalized Anxiety Disorder 7-Item Scale (GAD-7) was used to assess anxiety symptoms. Patients were asked to rate how often they had been bothered by the described symptoms over the last 2 weeks using a 4-point rating scale from 0 (not at all) to 3 (every day). Total scores range from 0 to 21, with higher scores reflecting higher severity levels of generalized anxiety disorder symptomology. ${ }^{25}$ Depressive symptoms were measured using the Patient Health Questionnaire-9 (PHQ9). This questionnaire comprises 9 items with scores between 0 and 27. Higher scores reflect higher severity levels of depressive symptoms. ${ }^{25,26}$ All patients completed the three questionnaires again from May 20 to 29, 2020.

During the study period, the nonintervention group received consultation and treatment procedures that are inherent to routine clinical practice.

\section{Statistics}

SPSS 24.0 was used to analyze the data. The independent variables were group and time; the total QOLIE-31, GAD7 and PHQ-9 scores were used as dependent variables. Two-factor repeated measurement analysis of variance (ANOVA) of $2 \times 2$ was performed, with $\mathrm{P}<0.05$ indicating a significant difference. 


\section{Results}

\section{Study Population}

On February 15 to 25, 2020, there were 809 inpatients from January 2018 to January 2020 obtained through the electronic medical record query system. To eliminate the possible interference caused by other seizure types, we chose only FE, which did not arise from uniform epilepsy syndrome/ localization. Among the 809 inpatients, 134 PWE were FE and oral oxcarbazepine, but 25 patients were unwilling to participate in the study, 7 patients were unable to finish the questionnaire, 10 patients were under 14 years old, and 2 patients had a mental disorder. Finally, a total of 90 patients completed the questionnaires and were divided into two groups, with 45 patients in each group. We numbered them according to the time of admission, odd numbers into the intervention (IG) group and even numbers into the nonintervention (NIG) group. The patients ranged in age from 14 to 65 years, with a mean age of $39.91 \pm 15.57$

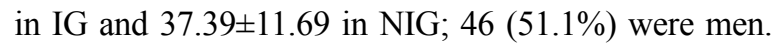

\section{Lives Affected by COVID-19 During the Follow-Up}

Up to 84 patients' lives were adversely affected, 43 (95.6\%) in IG and 41 (91.1\%) in NIG. Since the start of confinement, 43 (95.6\%) IG and 38 (84.4\%) NIG patients reported lifestyle changes, mainly reporting that their usual sleep schedules had changed. Fifteen (33.3\%) IG and 20 (44.4\%) NIG patients stated that their usual family income had decreased because of confinement-related restrictions; among them, 13 $(28.9 \%)$ IG and $10(22.2 \%)$ NIG stated that they were unemployed. There were no statistically significant differences in epilepsy-related or demographic factors between the two groups ( $p>0.05)$; for details, see Table 1 .

Table I General Information on the Two Groups of Patients (n, \%)

\begin{tabular}{|c|c|c|c|c|}
\hline General Information & IG & NIG & $\mathbf{p}$ & $\chi^{2} / t$ \\
\hline \multicolumn{5}{|l|}{ Gender: } \\
\hline Male & $24(53.3 \%)$ & $22(48.9 \%)$ & 0.673 & 0.178 \\
\hline Female & $21(46.7 \%)$ & $23(51.1 \%)$ & & \\
\hline Cerebrovascular diseases & $7(15.6 \%)$ & $9(20.0 \%)$ & 0.581 & 0.304 \\
\hline Skin disease & $\mathrm{I}(2.2 \%)$ & $0(0 \%)$ & 0.315 & 1.011 \\
\hline Alcoholism & $\mathrm{I}(2.2 \%)$ & $2(4.4 \%)$ & 0.557 & 0.345 \\
\hline Menstrual disorders & $\mathrm{I}(2.2 \%)$ & $\mathrm{I}(2.2 \%)$ & 1.000 & 0.000 \\
\hline Urinary infection & $\mathrm{I}(2.2 \%)$ & $0(0 \%)$ & 0.315 & 1.011 \\
\hline Hypertension & $5(11.1 \%)$ & $7(15.6 \%)$ & 0.535 & 0.385 \\
\hline Hyperlipidemia & $2(4.4 \%)$ & $0(0 \%)$ & 0.153 & 2.045 \\
\hline The EEG was normal & $25(55.6 \%)$ & $2 \mathrm{I}(46.7 \%)$ & 0.399 & 0.711 \\
\hline Difficult to buy AEDs & $9(20 \%)$ & II (24.4\%) & 0.612 & 0.257 \\
\hline Patients with seizures in I M & $3(6.7 \%)$ & $4(8.9 \%)$ & 0.694 & 0.155 \\
\hline Seek medical advice after the seizure & $0(0 \%)$ & $\mathrm{I}(2.2 \%)$ & 0.315 & 1.011 \\
\hline Unemployment & $13(28.9 \%)$ & $10(22.2 \%)$ & 0.468 & 0.526 \\
\hline Patients with life changed & $43(95.6 \%)$ & $4 \mathrm{l}(91.1 \%)$ & 0.398 & 0.714 \\
\hline Transportation mode & $40(88.9 \%)$ & $38(84.4 \%)$ & 0.535 & 0.385 \\
\hline Life-style & $43(95.6 \%)$ & $35(77.8 \%)$ & 0.013 & 6.154 \\
\hline Income & $15(33.3 \%)$ & $20(44.4 \%)$ & 0.280 & 1.169 \\
\hline Social-style & $28(62.2 \%)$ & $15(33.3 \%)$ & 0.006 & 7.526 \\
\hline Age range & $14-65$ & $14-59$ & 0.542 & 0.615 \\
\hline Course of disease & $1-32$ & $1-34$ & 0.889 & -0.141 \\
\hline
\end{tabular}

Abbreviations: IG, intervention group; NIG, nonintervention group; EEG, electroencephalogram; AEDs, antiepileptic drugs; M, month. 
Table 2 Two-Way Repeated Measures ANOVA of QOLIE-3I, PHQ-9 and QAD-7

\begin{tabular}{|c|c|c|c|c|c|}
\hline Source of Variation & SS & df & MS & $\mathbf{F}$ & $\mathbf{p}$ \\
\hline \multicolumn{6}{|l|}{ Interaction on QOLIE-3I } \\
\hline Group & 276.793 & 1 & 276.793 & 3.228 & 0.076 \\
\hline Error (Group) & 7545.294 & 88 & 85.742 & & \\
\hline Time & 818.987 & 1 & 818.987 & 50.226 & 0.000 \\
\hline Group * time & 276.644 & 1 & 276.644 & 16.966 & 0.000 \\
\hline Error (time) & 1434.936 & 88 & 16.306 & & \\
\hline \multicolumn{6}{|l|}{ Interaction on PHQ-9 } \\
\hline Group & 10.272 & 1 & 10.272 & 2.928 & 0.091 \\
\hline Error (Group) & 308.756 & 88 & 3.509 & & \\
\hline Time & 36.450 & 1 & 36.450 & 64.669 & 0.000 \\
\hline Group * time & 8.450 & 1 & 8.450 & 14.992 & 0.000 \\
\hline Error (time) & 49.600 & 88 & 0.564 & & \\
\hline \multicolumn{6}{|l|}{ Interaction on $\mathrm{QAD}-7$} \\
\hline Group & 15.606 & I & 15.606 & 1.286 & 0.260 \\
\hline Error (Group) & 1067.844 & 88 & 12.135 & & \\
\hline Time & 2212.006 & 1 & 2212.006 & 1086.118 & 0.000 \\
\hline Group * time & 12.272 & 1 & 12.272 & 6.026 & 0.016 \\
\hline Error (time) & 179.222 & 88 & 2.037 & & \\
\hline
\end{tabular}

Note: *Interaction between time and group in the ANOVA.

Abbreviations: ANOVA, analysis of variance; SS, stdev square; df, degree of freedom; MS, mean square; QOLIE-3I, Quality Of Life In Epilepsy-3I; PHQ-9, Patient Health Questionnaire-9; GAD-7, Generalized Anxiety Disorder 7-Item Scale.

\section{Epilepsy-Related Characteristics During the Follow-Up}

The seizure type was all focal onsets. There were no one report changes of seizure type during the follow-up. All patients were treated with Oxcarbazepine at baseline. Twenty patients had difficulty purchasing AEDs, 9 (20\%) in IG and 11 (24.4\%) in NIG. 2 patients $(4.4 \%)$ in IG and 4 patients $(8.9 \%)$ in NIG change Oxcarbazepine to Carbamazepine 3 month later. During the outbreak, 3 patients in IG $(6.7 \%)$ and 4 patients in NIG $(8.9 \%)$ experienced seizures in the last month. Among those only 1 patient $(2.2 \%)$ consulted the emergency department. Nobody showed poor response to AEDs. Twenty $(22.2 \%)$ reported difficulties obtaining their medication supply, $9(20 \%)$ in IG and $11(24.4 \%)$ in NIG because they could not leave their home, and the mail by post had stopped. Because the two drugs (carbamazepine and phenobarbital) are currently distributed free of charge by the Chinese government, they could easily obtain these two kinds of AEDs in local grassroots hospitals, so we suggested that they temporarily switch.

\section{The IG Group QOLIE-3I Scores Improved More Than the NIG Group}

After the 3-month follow-up period, the total QOLIE-31 score was significantly improved compared to baseline in both groups $(\mathrm{p}<0.001)$. The improvement in QOLIE-31 for the IG was greater than that for the NIG $(p<0.05)$ (Figure 1A). The results of ANOVA showed that the interaction between group and time on the QOLIE-31 was significant, $F(1,88)=16.996, p<0.001$; the main effect of the group on the QOLIE-31 was not significant, $\mathrm{F}(1,88)=3.228, \mathrm{p}>0.05$; there was no significant difference in the QOLIE-31 between the IG and the NIG; the main effect of time on the QOLIE-31 was significant, $\mathrm{F}(1,88)=50.226, \mathrm{p}<0.001$; the QOLIE-31 was significantly different between baseline and 3 months later; 3 months later, the QOLIE-31 in the IG and the NIG were significantly higher than the baseline, and the IG improved more than the NIG. For details, see Table 2. 
A

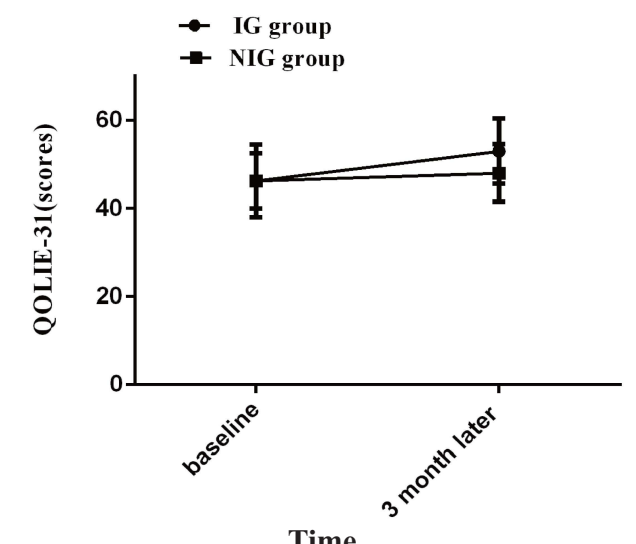

C

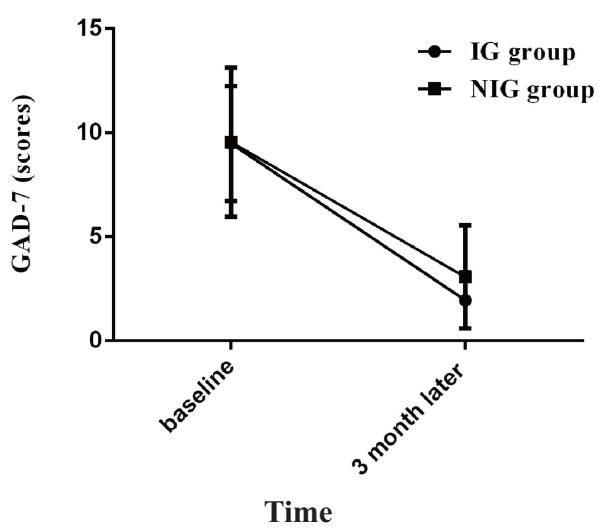

B

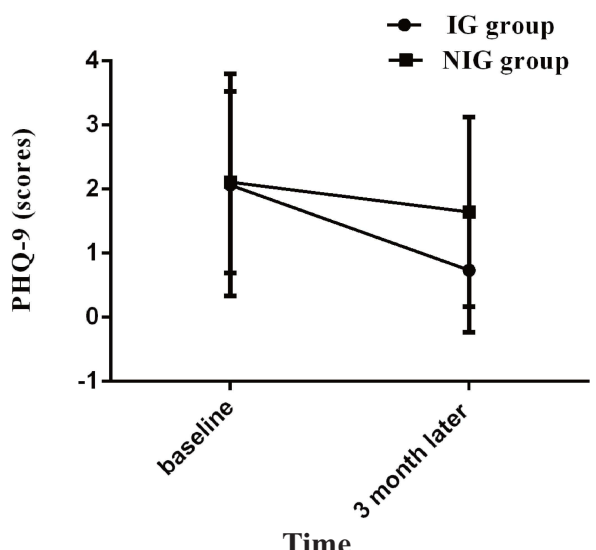

Figure I (A) Comparison of QOLIE-3I scores between the two groups before and after the intervention. (B) Comparison of PHQ-9 scores between the two groups before and after the intervention. (C) Comparison of GAD-7 scores between the two groups before and after the intervention.

\section{The Results of ANOVA on the PHQ-9}

The ANOVA results showed that the interaction between group and time on the PHQ-9 was significant, $F(1,88)=$ $14.992, \mathrm{p}<0.001$. The main effect of time on the PHQ-9 was significant, $F(1,88)=64.669, p<0.001$; the main effect of the group on PHQ-9 was not significant, $\mathrm{F}(1,88)=$ 2.928, $\mathrm{p}>0.05$; the difference between PHQ-9 in the IG and the NIG was not significant; the scores of the PHQ-9 questionnaire of the IG and the NIG were significantly lower than the baseline after 3 months, and the IG decreased more than the NIG (For details, see Table 2 and Figure 1B).

\section{The Results of the ANOVA on the QAD-7}

The results of ANOVA showed that the interaction between group and time was significant, $\mathrm{F}(1,88)=$ $6.026, \mathrm{p}<0.001$; the main effect of the group on QAD-7 was not significant, $\mathrm{F}(1,88)=1.286, \mathrm{p}>0.05$, the difference between QAD-7 in the IG and the NIG was not significant; the main effect of time on the QAD-7 was significant, $F(1,88)=1086.118, \mathrm{p}<0.001$, the scores on the QAD-7 questionnaire of the IG and the NIG were significantly lower than the baseline after three months, and the IG decreased more than the NIG (For details, see Table 2 and Figure 1C).

\section{Discussion}

The main purpose of this study was to observe the influence of telemedicine and humanistic care on the quality of life and mental health of PWE in northeastern China during the COVID-19 crisis.

This study found that during the COVID-19 pandemic, the lives of more than $90 \%$ of PWE were adversely affected, such as unemployment, income reduction and lifestyle changes. The results of ANOVA showed that the interaction between group and time on the QOLIE-31 was 
significant; the main effect of the group on the QOLIE-31 was not significant; the main effect of time on the QOLIE31 was significant; and the QOLIE-31 was significantly different between baseline and 3 months later. These results indicate that in our study, over time, the quality of life in both groups were improved. This because the interaction is the principal test of the hypothesis under investigation. ${ }^{27}$ A study in Hong Kong uniquely adopt an empowerment-based approach to equip patients as active agents through an integrated care model that comprehensively addresses their needs, better changes in medication adherence, anxiety, depression and health-related quality of life after the intervention. ${ }^{28}$ The Feldenkrais method is a mind-body therapy through movement lessons which are verbally guided explorations of movement. It aims to promote increased functioning in contexts in which the entire body cooperates in the execution of movements. ${ }^{29}$ A Mata analysis show that behaviors positively associated with patients health outcomes included empathy, reassurance and support, various patient-centered questioning techniques, explanations, positive reinforcement, psychosocial talk, time in health education and information sharing, friendliness and so on. ${ }^{30}$ Our results are consistent with these studies, especially during the pandemic, and help to improve the health of patients.

The baseline and later follow-up QOLIE-31 scores of PWE in northeastern China were significantly lower than the global average during the pandemic. ${ }^{31}$ However, we checked the QOLIE-31 scores in some parts of China, which also lower than the global average, including $42.15 \pm 17.63$ in rural areas of Tibet in $2007,{ }^{32} 55 \pm$ 12.5 in rural areas of Henan Province in 2020. ${ }^{33}$ Research shows that the quality of life of PWE in China is positively correlated with educational income and negatively correlated with the course of disease, condition and seizure frequency. ${ }^{34}$ The income level in northeastern China is at lower level for the country, the compliance of PWE with treatment is poor, and the epilepsy control rate is low. In addition, we observed that as time went on, the scores of the NIG group also increased because as the information became increasingly clear, an effective isolation policy was implemented by the country. Although the information was gradually clearer, the economic recovery of northeastern China was poor, and the unemployment rate remained high. In addition, the QOLIE-31 score at baseline and later follow-up in this study was obtained at the most serious time of COVID-19, while the global average score of the QOLIE-31 was obtained under normal circumstances. It should be determined the average score in northeastern China by reinvestigation during a relatively stable period.

The mean baseline scores on the GAD7 were quite high, with $>7$ considered a useful cut point for evaluating the risk of clinically significant disorders, and the change in the GAD-7 scores (in both groups) was substantial. ${ }^{35}$ Conversely, the PHQ-9 scores were notably low. This is slightly surprising given the high prevalence of mood disorders in epilepsy. The optimal cutoff for the PHQ-9 was similar $(\geq 5)$ for mild and moderate depression and $\geq 7$ for severe depression. Compared with other studies, the low prevalence in our study might be explained by the differences in methodology, length of follow-up, and sample size. Firstly, it is not excluded that the form of telephone follow-up affects the results to a certain extent. Patients may have a stronger sense of shame. Some patients are working or surrounded by others during telephone follow-up. The introverted character of Chinese people makes them more conservative in answering depression related questions. The effect is not as good as face-to-face conversation. Anxiety is a widespread and consistent emotion in the epidemic, and patients are more willing to share this emotion with doctors in telephone interviews. Secondly, our follow-up time was relatively short, and some possible changes were not reflected in time. And we also need to increase the sample size.

Telehealth has many advantages during this time of social distancing. Leveraging the clinical support team can create a virtual epilepsy clinic that provides appropriate lifestyle and seizure safety counseling while testing and identifying psychiatric comorbidities. ${ }^{36}$ Images and EEG studies can be reviewed with the family using video conference applications. Referrals to epilepsy centers can be initiated remotely. ${ }^{13}$ Telemedicine can play a role in the follow-up care of PWE, reduce patient costs, and improve patient satisfaction. ${ }^{11}$ At the same time, there are also limitations to using telemedicine. The struggle for digital inclusion might result in the marginalization of non-technology users including the elderly and patients with lower socioeconomic status. The majority of child neurologists found it inappropriate to evaluate a child less than 1 year of age via telemedicine. ${ }^{13}$ A study on the reasons why neurologists refuse telemedicine shows that specific reasons include lack of time, fear of excessive organizational burden, concern about unqualified medical equipment, and reimbursement of telemedicine expenses. Neurologists in rural areas prefer to request telemedicine 
consultation before referral, which is consistent with the previous practice of our center. ${ }^{37}$ Before the epidemic, our center only conducted video connection consultations with experts in Beijing for difficult cases. Due to the emergence of the epidemic, telemedicine has become more prominent. ${ }^{38-40}$ However, the knowledge of the patients and the economic level of northeastern China do not allow for large-scale telemedicine. Patients seem to rely more on face-to-face communication because of low knowledge about telemedicine. Second, there is almost no infrastructure for telemedicine in this region. During public health outbreaks, clinicians and caregivers should focus not only on seizure control but also on the mental health of PWE. ${ }^{5}$ Our telemedicine paid more attention to the emotional and life changes of patients through questionnaires but it lacked physical examination, comorbidity screening and prevention, and could not provide EEG examinations. For some patients whose city was strictly isolated, telemedicine became the only means of doctor-patient connection and it has played an important role.

At present, there is no unified and detailed standard for compassionate care. To make our sympathy and care for all patients as consistent as possible, we set a simple standard set of questions suitable for China at that time (the standard here means that all patients were asked the same questions). These questions are not questionnaires in the strict sense, so there is no Cronbach's alpha.

There are still some deficiencies in this experiment. Many confounders may influence the results. First, the sample size was relatively small and our research period was relatively short. It is better to increase the number of patients and prolong the observation period in the future. Second, we did not perform a correlation analysis between the general condition, quality of life and emotional changes. The two groups of patients in the course of disease time, comorbidity, family situation, and other aspects of the difference cannot be completely consistent; in the future, the two groups should be assessed as much as possible with consistent research. In addition, we did not identify whether the intervention helped to relieve epilepsy-related comorbidities since the pandemic occurred suddenly. The clinical background of this outbreak occurred without other options, which is difficult to replicate. Medicaid coverage and insurance reimbursement for telehealth played a pivotal role in the crisis. The Chinese government paid for all treatment fees for COVID-19, so the study did not consider this situation, but around the world, we should consider this.
Although the period of this study was relatively short, compassionate care had a positive impact on the quality of life and mood of epilepsy patients in northeastern China. In June 2020, the local epidemic situation significantly improved, and it was possible for PWE to purchase drugs and obtain rechecks. Our center still chose nurses to continue monthly compassionate care for patients. Due to professional and time constraints, the living effects caused by the epidemic have resolved. The questionnaire model used in this study was only partially preserved (mainly Part 3). Although this means our follow-up was less intensive, the patients' satisfaction with our follow-up was $100 \%$. This suggests we can appropriately reduce the frequency of follow-up but increase the content of follow-up to improve the quality of follow-up, such as multidisciplinary consultation; increase compassionate care for patients with mental disorders; increase investment in inspection facilities in grassroots hospitals; and increase volunteer service projects worthy of further study.

\section{Conclusions}

Our study found that during the COVID-19 outbreak, when patients were in a lockdown, telemedicine and humanistic care were effective and successful in patients with epilepsy in northern China. It was a valid method to decrease anxiety and depression and improve the patients' quality of life. Further research is needed in the field of compassionate care methods for patients with epilepsy.

\section{Abbreviations}

COVID-19, Coronavirus Disease 2019; QOLIE-31, Quality of Life in patients with Epilepsy-31 items; GAD7, Generalized Anxiety Disorder-7 items; PHQ-9, Patient Health Questionnaire-9 items; EEG, electroencephalogram; ILAE, International League Against Epilepsy.

\section{Data Sharing Statement}

The datasets used and/or analyzed during the current study are available from the corresponding author upon reasonable request.

\section{Ethics Approval and Consent to Participate}

The experimental protocol was established according to the ethical guidelines of the Helsinki Declaration and was approved by the Human Ethics Committee of Jiamusi Central Hospital. Oral informed consent was obtained from individual or guardian participants. 


\section{Consent for Publication}

Not applicable.

\section{Acknowledgments}

Deshuang Tao and Tangwu Zhong are co-first authors for this study.

\section{Author Contributions}

All authors made a significant contribution to the work reported, whether that is in the conception, study design, execution, acquisition of data, analysis and interpretation, or in all these areas; took part in drafting, revising or critically reviewing the article; gave final approval of the version to be published; have agreed on the journal to which the article has been submitted; and agree to be accountable for all aspects of the work.

\section{Disclosure}

The authors report no conflicts of interest in this work.

\section{References}

1. Cucinotta D, Vanelli M. WHO declares COVID-19 a pandemic. Acta Biomed. 2020;91(1):157-160. doi:10.23750/abm.v91i1.9397

2. Tu H, Tu S, Gao S, et al. Current epidemiological and clinical features of COVID-19; a global perspective from China. $J$ Infect 2020;81(1):1-9. doi:10.1016/j.jinf.2020.04.011

3. Thorpe J, Ashby S, Hallab A, et al. Evaluating risk to people with epilepsy during the COVID-19 pandemic: preliminary findings from the COV-E study. Epilepsy Behav. 2021;115:107658. doi:10.1016/j. yebeh.2020.107658

4. French JA, Brodie MJ, Caraballo R, et al. Keeping people with epilepsy safe during the COVID-19 pandemic. Neurology. 2020;94 (23):1032-1037. doi:10.1212/WNL.0000000000009632

5. Hao X, Zhou D. Severe psychological distress among patients with epilepsy during the COVID-19 outbreak in southwest China. Epilepsia. 2020;61(6):1166-1173. doi:10.1111/epi.16544

6. Koh M-Y, Lim K-S, Fong S-L, et al. Impact of COVID-19 on quality of life in people with epilepsy, and a multinational comparison of clinical and psychological impacts. Epilepsy Behav. 2021;117: 107849. doi:10.1016/j.yebeh.2021.107849

7. McKenna MC, Al-Hinai M, Bradley D, et al. Patients' experiences of remote neurology consultations during the COVID-19 pandemic. Eur Neurol. 2020;83(6):622-625. doi:10.1159/000511900

8. Craig J, Patterson V. Introduction to the practice of telemedicine. $J$ Telemed Telecare. 2005;11(1):3-9. doi:10.1177/1357633X050110 0102

9. Hong Z, Li N, Li D. Telemedicine during the COVID-19 pandemic: experiences from Western China. Journal of Medical Internet Research. 2020;22(5):e19577. doi:10.2196/19577

10. Patterson V. Neurological telemedicine in the COVID-19 era. Nat Rev Neurol. 2021;17(2):73-74. doi:10.1038/s41582-020-00438-9

11. Ahmed SN, Mann C, Sinclair DB, et al. Feasibility of epilepsy follow-up care through telemedicine: a pilot study on the patient's perspective. Epilepsia. 2008;49(4):573-585. doi:10.1111/j.15281167.2007.01464.x
12. Tailby C, Collins AJ, Vaughan DN, et al. Teleneuropsychology in the time of COVID-19: the experience of The Australian Epilepsy Project. Seizure. 2020;83:89-97. doi:10.1016/j.seizure.2020.10.005

13. Sattar S, Kuperman R. Telehealth in pediatric epilepsy care: a rapid transition during the COVID-19 pandemic. Epilepsy Behav. 2020;111:107282. doi:10.1016/j.yebeh.2020.107282

14. von Wrede R, Moskau-Hartmann S, Baumgartner T, et al. Counseling of people with epilepsy via telemedicine: experiences at a German tertiary epilepsy center during the COVID-19 pandemic. Epilepsy Behav. 2020;112:107298. doi:10.1016/j.yebeh.2020.107298

15. Flocke SA, Miller WL, Crabtree BF. Relationships between physician practice style, patient satisfaction, and attributes of primary care. $J$ Fam Pract. 2002;51(10):835-840.

16. Heyland DK, Cook DJ, Rocker GM, et al. Defining priorities for improving end-of-life care in Canada. Cmaj. 2010;182(16):E747-52. doi:10.1503/cmaj.100131

17. Puchalski CM, Vitillo R, Hull SK, et al. Improving the spiritual dimension of whole person care: reaching national and international consensus. J Palliat Med. 2014;17(6):642-656. doi:10.1089/jpm.2014.9427

18. van der Cingel M. Compassion in care: a qualitative study of older people with a chronic disease and nurses. Nurs Ethics. 2011;18 (5):672-685. doi:10.1177/0969733011403556

19. Bray L, O'Brien MR, Kirton J, et al. The role of professional education in developing compassionate practitioners: a mixed methods study exploring the perceptions xof health professionals and pre-registration students. Nurse Educ Today. 2014;34(3):480-486. doi:10.1016/j.nedt.2013.06.017

20. Renzi S, Fallanca F, Zangrillo A, et al. Caring with compassion during COVID-19. Palliat Support Care. 2020;18(4):403-404. doi:10.1017/S1478951520000577

21. Stimell-Rauch M, Szalay LR, Bregstein J. Telehealth and humanism: unexpected partnership forged during COVID-19. Emerg Med J. 2020;37(9):591-592. doi:10.1136/emermed-2020-209989

22. McNairy M, Bullington B, Bloom-Feshbach K. Searching for human connectedness during COVID-19. J Gen Intern Med. 2020;35 (10):3043-3044. doi:10.1007/s11606-020-06082-9

23. Scheffer IE, Berkovic S, Capovilla G, et al. ILAE classification of the epilepsies: position paper of the ILAE Commission for Classification and Terminology. Epilepsia. 2017;58(4):512-521. doi:10.1111/epi.13709

24. Martinović Ž, Milovanović M, Tošković O, et al. Psychometric evaluation of the Serbian version of the Quality of Life in Epilepsy Inventory-31 (QOLIE-31). Seizure. 2010;19(8):517-524. doi:10.10 16/j.seizure.2010.07.012

25. Garabiles MR, Lao CK, Yip P, et al. Psychometric Validation of PHQ-9 and GAD-7 in Filipino Migrant Domestic Workers in Macao (SAR), China. J Personal Assess. 2019;102:1-12.

26. Moyer DN, Connelly KJ, Holley AL. Using the PHQ-9 and GAD-7 to screen for acute distress in transgender youth: findings from a pediatric endocrinology clinic. J Pediatr Endocrinol Metab. 2019;32(1):71-74. doi:10.1515/jpem-2018-0408

27. Garrigós-Pedrón M, La Touche R, Navarro-Desentre P, et al. Effects of a physical therapy protocol in patients with chronic migraine and temporomandibular disorders: a randomized, single-blinded, clinical trial. J Oral Facial Pain Headache. 2018;32(2):137-150. doi:10.11 607/ofph.1912

28. Li PWC, Yu DSF, Yan BBY. A nurse-coordinated integrated care model to support decision-making and self-care in patients with atrial fibrillation: a study protocol. $J$ Adv Nurs. 2019;75(12):3749-3757. doi:10.1111/jan.14164

29. Paolucci T, Zangrando F, Iosa M, et al. Improved interoceptive awareness in chronic low back pain: a comparison of Back school versus Feldenkrais method. Disabil Rehabil. 2017;39(10):994-1001. doi:10.1080/09638288.2016.1175035

30. Beck RS, Daughtridge R, Sloane PD. Physician-patient communication in the primary care office: a systematic review. $J$ Am Board Fam Pract. 2002;15(1):25-38. 
31. Wiebe S, Matijevic S, Eliasziw M, Derry PA. Clinically important change in quality of life in epilepsy. $J$ Neurol Neurosurg Psychiatry. 2002;73(2):116-120. doi:10.1136/jnnp.73.2.116

32. Zhao Y. Analysis of natural history, the Quality of life in Tibetan patients with Convulsive Epilepsy and prevalence in rural Tibet:an initial survey.[dissertation]. Sichuan University; 2007.

33. Huang S, Zheng C, Wang M, Tan Z. Analysis of quality of life and treatment status of epilepsy patients in rural areas of Henan Province. Journal of Clinical Internal Medicine. 2020;5:375-376. Chinese.

34. Zhao Y, Wu H, Li J, et al. Quality of life and related factors in adult patients with epilepsy in China. Epilepsy Behav. 2011;22(2):376-379. doi:10.1016/j.yebeh.2011.07.025

35. Micoulaud-Franchi J-A, Lagarde S, Barkate G, et al. Rapid detection of generalized anxiety disorder and major depression in epilepsy: validation of the GAD-7 as a complementary tool to the NDDI-E in a French sample. Epilepsy Behav. 2016;57(Pt A):211-216. doi:10. 1016/j.yebeh.2016.02.015

36. Lau J, Knudsen J, Jackson H, et al. Staying Connected In The COVID-19 Pandemic: telehealth At The Largest Safety-Net System In The United States. Health Aff (Millwood). 2020;39(8):1437-1442. doi:10.1377/hlthaff.2020.00903
37. Zöllner JP, Noda AH, McCoy J, et al. [Attitudes and barriers towards telemedicine in epilepsy care: a survey among neurological practices]. Z. Epileptol. 2021:1-6. German. doi:10.1007/s10309-021-00417-0

38. Shaikh AG, Mitoma H, Manto M. Cerebellar scholars' challenging time in COVID-19 pandemia. Cerebellum. 2020;19(3):343-344. doi:10.1007/s12311-020-01131-9

39. Conde-Blanco E, Centeno M, Tio E, et al. Emergency implementation of telemedicine for epilepsy in Spain: results of a survey during SARS-CoV-2 pandemic. Epilepsy Behav. 2020;111:107211. doi:10.1016/j.yebeh.2020.107211

40. Kossoff EH, Turner Z, Adams J, et al. Ketogenic diet therapy provision in the COVID-19 pandemic: dual-center experience and recommendations. Epilepsy Behav. 2020;111:107181. doi:10.1016/j. yebeh.2020.107181
Journal of Multidisciplinary Healthcare

\section{Publish your work in this journal}

The Journal of Multidisciplinary Healthcare is an international, peerreviewed open-access journal that aims to represent and publish research in healthcare areas delivered by practitioners of different disciplines. This includes studies and reviews conducted by multidisciplinary teams as well as research which evaluates the results or conduct of such teams or healthcare processes in general. The journal

\section{Dovepress}

covers a very wide range of areas and welcomes submissions from practitioners at all levels, from all over the world. The manuscript management system is completely online and includes a very quick and fair peer-review system. Visit http://www.dovepress.com/testimonials. php to read real quotes from published authors. 